ORGANIC LEDS

\section{DNA benefits}

Sci. Rep. 4, 7105 (2014)

The nucleic acid bases that form DNA can serve as a useful hole-transport (electron blocking) layer in organic light emitting diodes (OLEDs), according to scientists in the USA. Eliot Gomez and co-workers report that because adenine and thymine can be thermally evaporated, they can be easily incorporated into the fabrication of OLEDs made by vacuum deposition and offer efficient hole-transport to the light emitting layer. Green phosphorescent OLEDs featuring adenine as a hole-transport layer achieved a peak current efficiency of $48 \mathrm{~cd} \mathrm{~A}^{-1}$ and a luminance of $93,000 \mathrm{~cd} \mathrm{~m}^{-2}$, and OLEDs using thymine achieved $76 \mathrm{~cd} \mathrm{~A}^{-1}$ and $132,000 \mathrm{~cd} \mathrm{~m}^{-2}$. In contrast, a reference device using the conventional holetransport material NPB yielded values of $37 \mathrm{~cd} \mathrm{~A}^{-1}$ and $113,000 \mathrm{~cd} \mathrm{~m}^{-2}$. The researchers believe the advantageous performance of the nucleic acid bases is due to their large highest occupied molecular orbital energy level, which helps create a more balanced electronhole ratio within the device. The low cost and ease of fabrication and processing of natural charge transport layers could make them an attractive material choice in the future for use in optoelectronic devices.

\section{LASER DIODES}

\section{Linewidth reduction}

Appl. Phys. Express 7, 122701 (2014)

An optical scheme for markedly reducing the linewidth of a semiconductor laser diode has been reported by Japanese scientists at Tohoku University and the NTT Device Technology Laboratories. Laser sources with a narrow linewidth are required for coherent optical communication schemes, such as those that use quadrature amplitude modulation. The technique relies on coherent optical negative feedback from a Fabry-Pérot etalon that is coupled to a $1,550 \mathrm{~nm}$ distributed-feedback laser diode (DFB-LD) via a Selfoc microlens. As the optical frequency of the DFB-LD increases or decreases with respect to its operation point, the reflectivity of the etalon changes, providing optical negative feedback that 'pulls' the laser back towards a central single frequency of operation. Konosuke Aoyama and co-workers report reducing the linewidth of the laser diode from $6.4 \mathrm{MHz}$ to $6.5 \mathrm{kHz}$ as well as a $30 \mathrm{~dB}$ reduction in the power spectral density of frequency noise for frequencies below $100 \mathrm{MHz}$.

$O G$

\section{QUANTUM INFORMATION}

\section{One-way quantum computer}

Phys. Rev. Lett. 113, 200501 (2014)

Simon's problem is of great importance in quantum algorithm design as it provides a clear gap between the classical and quantum times required to perform a calculation. However, it has never been experimentally realized in any physical system. Now, Mark Tame and co-workers in South Africa and the UK have demonstrated a one-way quantum algorithm for solving Simon's problem using a multipartite entangled state of photons. A Ti:sapphire laser with a wavelength of $724.5 \mathrm{~nm}$ pumped two birefringent photonic-crystal fibres (PCFs) to generate correlated photons with wavelengths of $626 \mathrm{~nm}$ and $860 \mathrm{~nm}$ through spontaneous four-wave mixing. Each PCF was arranged in a Sagnac loop closed using a polarization beam splitter to generate the
MID-INFRARED

Intraband quantum dots
ACS Nano 8, 11707-11714 (2014) five-qubit entangled cluster state plus an additional qubit. The algorithm was executed by measuring the relative populations and the polarization states. The team repeated the algorithm for the two-qubit version of Simon's Problem a number of times to obtain the success probabilities. The average runtime was around 2 iterations, whereas it was $8 / 3$ iterations on average for the classical analogue, thus experimentally demonstrating the existence of a runtime gap.

\section{LIGHT-MATTER COUPLING}

\section{Dynamic observation}

Optica 1, 276-280 (2014)

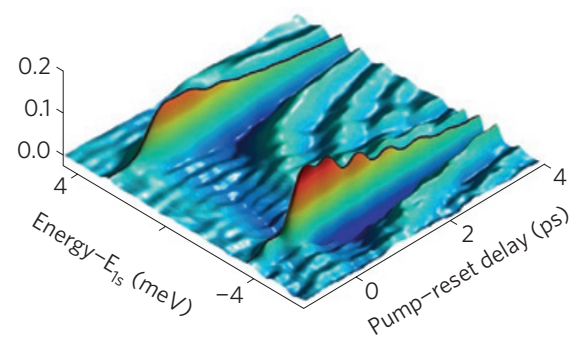

Although strong light-matter interactions leading to the formation of polaritons are widely studied in a variety of systems, the detailed dynamics are poorly understood. Now, Anthony David Jameson and colleagues at Oregon State University and the University of Arizona in the USA, and the Philipps-Universität in Marburg, Germany have proposed a so-called pump-reset technique for probing the time evolution of light-matter interaction in a semiconductor microcavity containing quantum wells. In this scheme, a pump pulse creates the polariton and a subsequent single-cycle $\mathrm{THz}$ reset pulse is used to switch off the coupling. The perturbation caused by the reset pulse is revealed in modulations of the pump-reflection spectrum and in pronounced polariton oscillations with increasing pump-reset delay. The observed oscillation period is $0.66 \mathrm{ps}$, as expected, which corresponds to the $6.3 \mathrm{meV}$ energy splitting between the two polaritons. The observed spectrotemporal interference pattern provides direct proof of the coherent character of the light-matter interaction dynamics. The authors anticipate that their scheme will be of considerable value in the field of plasmonic and organic devices, where light-matter coupling has attracted significant research interest. 